\title{
Roles of circular RNAs in the pathogenesis of intervertebral disc degeneration (Review)
}

\author{
ZHIDI LIN, FEIZHOU LU, XIAOSHENG MA, XINLEI XIA, FEI ZOU and JIANYUAN JIANG \\ Department of Orthopedics, Huashan Hospital, Fudan University, Shanghai 200040, P.R. China
}

Received May 19, 2021; Accepted July 26, 2021

DOI: $10.3892 / \mathrm{etm} .2021 .10655$

\begin{abstract}
Lower back pain (LBP) is an extremely common symptom and is recognized as a leading contributor to disability and disease burden globally. Intervertebral disc degeneration (IDD) represents a major cause of LBP. However, the molecular mechanisms involved in the pathogenesis of IDD remain unclear, and currently available treatments, including conservative and surgical options, fail to effectively delay, stop or reverse the progression of IDD. Circular RNAs (circRNAs) are a newly discovered group of covalently closed, single-stranded and endogenous non-coding RNAs. A growing body of research has revealed that a number of circRNAs are widely and aberrantly expressed in IDD tissues. Furthermore, they play important roles in the pathogenesis of IDD, including proliferation, apoptosis, senescence, mitophagy, inflammation and extracellular matrix metabolism, mainly by acting as sponges for microRNAs. The present review aims to summarize the current understanding on the mechanisms of circRNA-mediated regulation in IDD.
\end{abstract}

\section{Contents}

1. Introduction

2. Roles of circRNA in ECM metabolism

3. Roles of circRNA in cell proliferation and apoptosis

4. Roles of circRNA in inflammation

5. Roles of circRNA in senescence

6. Roles of circRNA in mitophagy

7. Limitations and future directions

8. Conclusion

Correspondence to: Dr Fei Zou or Professor Jianyuan Jiang, Department of Orthopedics, Huashan Hospital, Fudan University, 12 Wulumuqi Middle Road, Shanghai 200040, P.R. China

E-mail: zoufei@huashan.org.cn

E-mail: jjy@fudanspine.com

Key words: intervertebral disc degeneration, circular RNA, microRNA sponge, nucleus pulposus cell, extracellular matrix

\section{Introduction}

Lower back pain (LBP) is one of the most common symptoms and/or disorders globally, affecting people of all ages, and is now the number one cause of disability worldwide $(1,2)$. It has been reported that $\sim 84 \%$ of the general population suffers from LBP during their lifetime (3), and roughly $10 \%$ of them become chronically disabled (4). Due to the extremely high prevalence of LBP, it seriously harms society and the economy, placing a heavy burden on health care systems globally (5).

Intervertebral disc degeneration (IDD) is a major pathological contributor to LBP. The intervertebral disc is a complex fibrocartilaginous tissue that connects adjacent vertebral bodies and confers spinal mobility; it is also the largest avascular structure in the human body, consisting of the nucleus pulposus (NP), annulus fibrosus (AF) and cartilage endplate (CEP). Lacking a blood supply, disc cells retrieve oxygen and nutrients mainly by diffusion through CEPs. Thus, they have a limited capacity for self-repair after damage or degeneration (6). The extracellular matrix (ECM) is mainly synthesized and secreted by NP cells (NPCs) and is composed of type II collagen (collagen II) and aggrecan, helping to resist compression and maintain disc height. According to previous studies, IDD is mainly characterized by dysregulation of NPC survival, an imbalance between ECM anabolism and catabolism, and aberrant activation of the inflammatory response $(7,8)$.

As the molecular mechanisms of IDD are complicated and remain unclear, circular RNAs (circRNAs) have attracted growing attention in the past few years. circRNAs are a novel class of covalently closed, single-stranded and endogenous non-coding RNAs, unlike linear RNAs, without 5'-3' polarities and polyadenylation. Most circRNAs are produced from exons by a backsplicing mechanism in eukaryotes with cell type- and developmental stage-specific expression patterns (9). Compared with linear RNAs, circRNAs are generally more stable and resistant to RNase $\mathrm{R}$ digestion owing to their loop structure. circRNAs are abundant and evolutionarily conserved, and mainly localize to the cytoplasm. They exert important biological functions by acting as microRNA (miRNA/miR) sponges, which are also known as competing endogenous RNA (ceRNA) mechanisms, in the pathological process of various diseases (10).

Previous studies have shown that circRNAs play critical roles in various musculoskeletal diseases, including osteoarthritis, rheumatoid arthritis, osteoporosis, osteosarcoma, 
osteonecrosis, scoliosis and spinal cord injury (11-17). Recently, accumulating evidence has uncovered the significant differential expression of circRNAs between IDD and control groups. Gain-of-function, loss-of-function and rescue experiments were generally performed to investigate the biological functions and regulatory pathways of circRNAs. Luciferase reporter, pulldown and immunoprecipitation assays were performed to confirm the direct interactions among the molecules of signaling pathways of circRNAs $(18,19)$.

The present review summarizes the current evidence on the roles of circRNAs in the pathogenesis of IDD, including proliferation, apoptosis, senescence, mitophagy, inflammation and ECM metabolism (Table I).

\section{Roles of circRNA in ECM metabolism}

ECM degradation is a key contributor to the progression of IDD, leading to loss of compression resistance and disc height. Under pathological conditions, the balance between the synthesis and decomposition of the ECM is disturbed, which is characterized by decreased structural components (collagen II and aggrecan) and increased matrix-degrading enzymes [matrix metalloproteinase (MMP)-2, MMP-3, MMP-13, A disintegrin-like and metalloproteinase with thrombospondin type-1 motifs (ADAMTS)-4 and ADAMTS-5] (20-22).

Downregulated expression of circRNAs in ECM metabolism. Cheng et al (23) first elucidated the role of a specific circRNA in the pathological process of IDD. circVMA21 was significantly downregulated in IDD tissues compared with control tissues. Overexpression of circVMA21 reversed the imbalance between ECM anabolism and catabolism in NPCs after treatment with tumor necrosis factor- $\alpha$ (TNF- $\alpha$ ) and interleukin- $1 \beta$ (IL-1 $\beta$ ) in vitro. Luciferase reporter, pulldown, RNA immunoprecipitation and fluorescence in situ hybridization assays confirmed that circVMA21 directly binds with cytoplasmic miR-200c and subsequently upregulates the expression of the target mRNA, X linked inhibitor-of-apoptosis protein (XIAP).

Wang et al (24) found that circSEMA4B overexpression in NPCs attenuated the adverse effects on ECM synthesis induced by IL-1 $\beta$ treatment in vitro. It was further verified that circSEMA4B exerted positive functions by sponging miR-431 and subsequently upregulating the expression of secreted frizzled related protein 1 and glycogen synthase kinase-3 $\beta$, which are known as Wnt signaling-related factors. Similarly, it was reported that circERCC2 facilitated ECM anabolism in NPCs by sponging miR-182-5p and elevating the expression level of silent mating type information regulation 2 homolog 1 (SIRT1) (25).

Guo et al (26) suggested that circ-GRB10 enhanced NPC survival through the miR-328-5p-Erb-b2 receptor tyrosine kinase 2 (ERBB2) axis. Notably, another study by Guo et al (27) focused on circ-GRB10 to elucidate the upstream mechanisms. Forced expression of circ-GRB10 promoted phosphorylated-Erk $1 / 2$ expression and ameliorated ECM synthesis. RNA-binding protein FUS (FUS) showed strongly downregulated expression, while significant upregulation of miR-141-3p expression was observed in IDD samples. The positive effects induced by FUS overexpression could be counteracted by silencing circ-GRB10. In addition,
RNA immunoprecipitation demonstrated the direct interaction between FUS and circ-GRB10. Moreover, combined with further investigations and the results of the previous study (26), Guo et al (27) established the signaling circuitry of the circ-GBR10-miR-328-5p-ERBB2-Erk1/2 phosphorylation-miR-141-3p-FUS loop in the pathogenesis of IDD (Fig. 1).

Chen et al (28) confirmed that circGLCE attenuates ECM degradation by competitively absorbing miR-587 to upregulate signal transducing adaptor family member 1 expression. Huang et al (29) reported that circPKNOX1 facilitated ECM synthesis and restrained ECM decomposition. Dual luciferase assays indicated that circPKNOX1 directly interacts with miR-370-3p and subsequently upregulates KIAA0355 expression.

Upregulated expression of circRNAs in ECM metabolism. Wang et al (30) identified the significant upregulation of circ-4099 expression in NPCs after treatment with TNF- $\alpha$ in vitro. Overexpression of circ-4099 promoted ECM anabolism, which was rescued by miR-616-5p mimics. Further experiments revealed that circ-4099 ameliorates ECM synthesis through the miR-616-5p-SRY-related high mobility group-box gene 9 (Sox9) axis. It was reported that knockdown of circRNA_104670 increased ECM synthesis, which was counteracted by miR-17-3p inhibition (31). Luciferase reporter and enhanced green fluorescent protein/red fluorescent reporter assays demonstrated that circRNA_104670 aggravates ECM degradation by sponging miR-17-3p and upregulating MMP-2 expression. Cui and Zhang (32) showed that silencing circ_001653 in NPCs ameliorated ECM synthesis and inhibited ECM decomposition. It was further determined that circ_001653 sponges miR-486-3p, which targets cell migration-inducing hyaluronan binding protein (CEMIP) to regulate ECM metabolism.

In the study by Xiang et al (33), compression treatment rather than inflammatory cytokines (ICs) was applied to treated NPCs and induced IDD. Highly expressed in IDD samples and compression-treated NPCs, circRNA-CIDN could ameliorate the pro-catabolic effects in NPCs induced by compression in vitro and ex vivo. Luciferase reporter and RNA immunoprecipitation assays verified the direct binding between circRNA-CIDN and miR-34a-5p, and between miR-34a-5p and SIRT1. Rescue experiments indicated that circRNA-CIDN overexpression blocked the pro-catabolic effects of the miR-34a-5p mimic, while knockdown of SIRT1 impaired the protective effects of circRNA-CIDN in compression-treated NPCs. circRNA-CIDN therefore alleviates ECM catabolism induced by compression through the miR-34a-5p-SIRT1 pathway. Guo et al $(34,35)$ reported that both circ-FAM169A and circ-TIMP2 shift ECM homeostasis towards catabolism in NPCs by functioning as ceRNAs through miR-583- $\beta$-transducin repeat containing (BTRC) and miR-185-5p-MMP-2 signaling, respectively.

Xiao et al (36) focused on endplate chondrocytes instead of NPCs and found that circRNA_0058097 expression was upregulated in endplate chondrocytes after treatment with intermittent cyclic tension in vitro. Tension treatment decreased collagen II, aggrecan and Sox 9 expression, and changed the cell shape in endplate chondrocytes. Further assays showed that circRNA_0058097 negatively regulated 


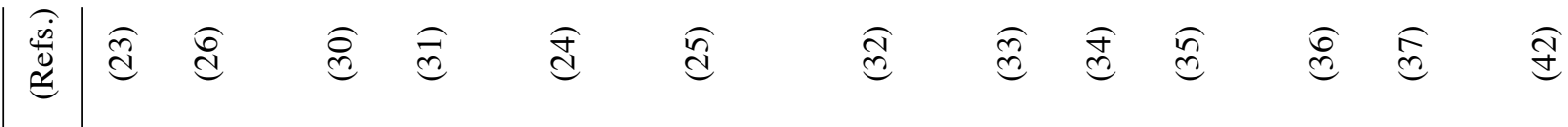

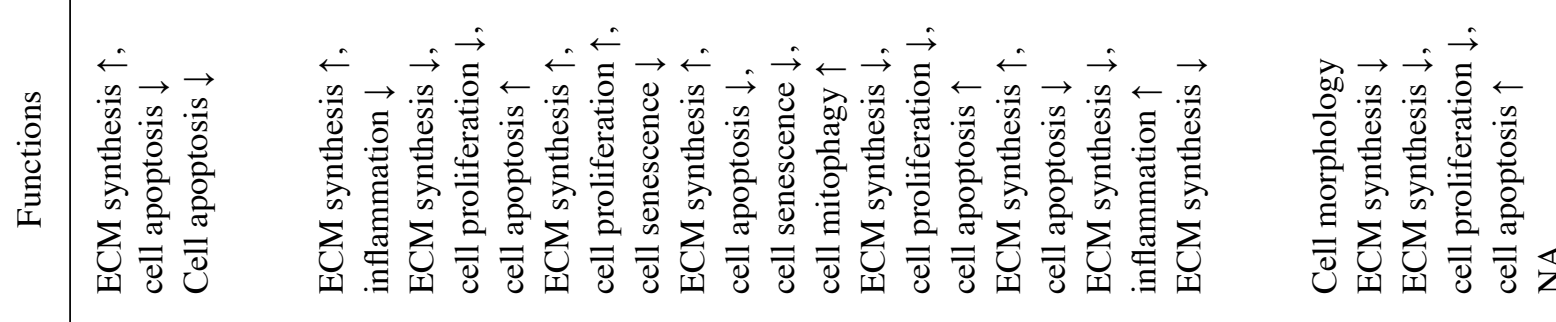

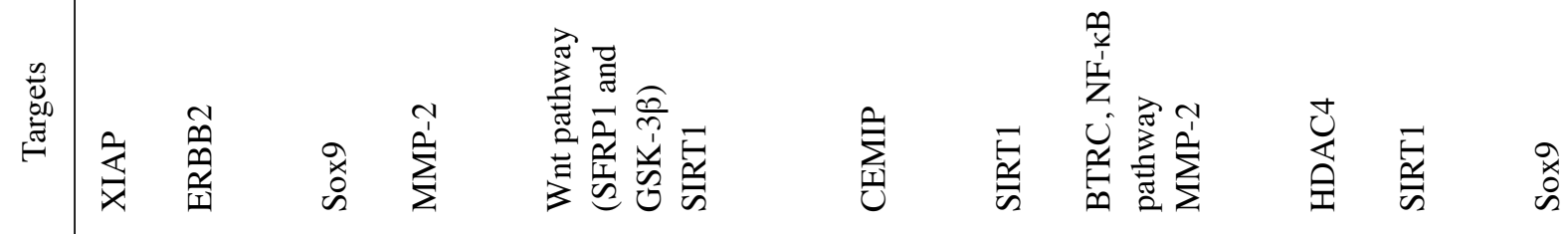

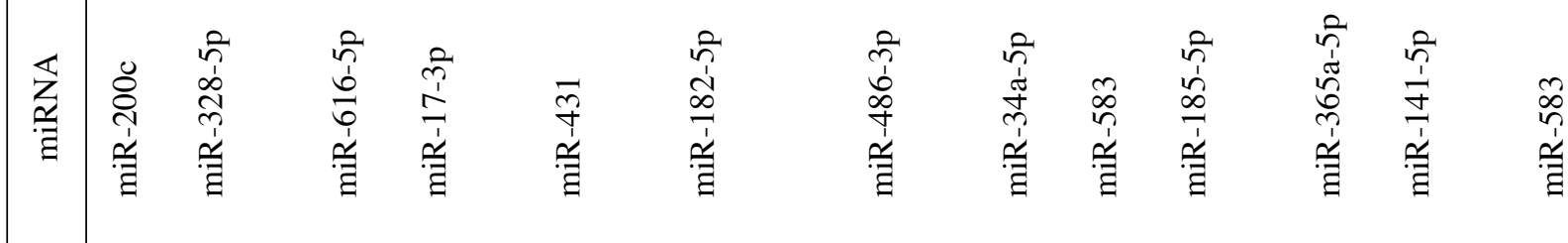

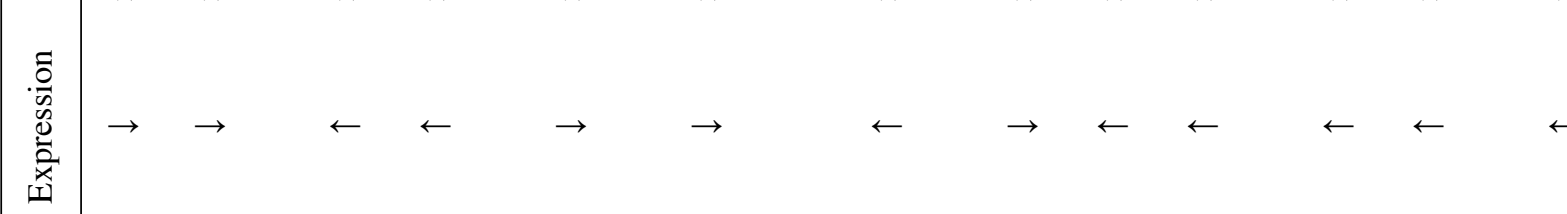

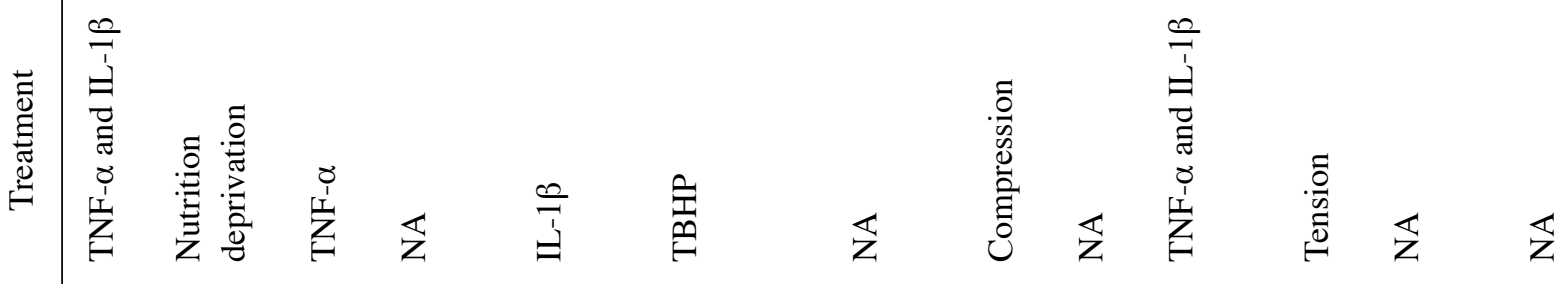

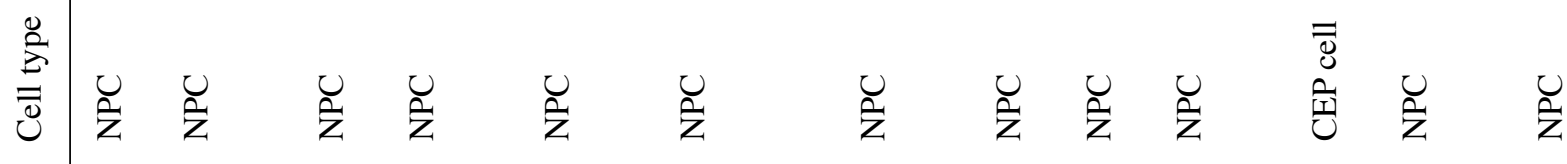

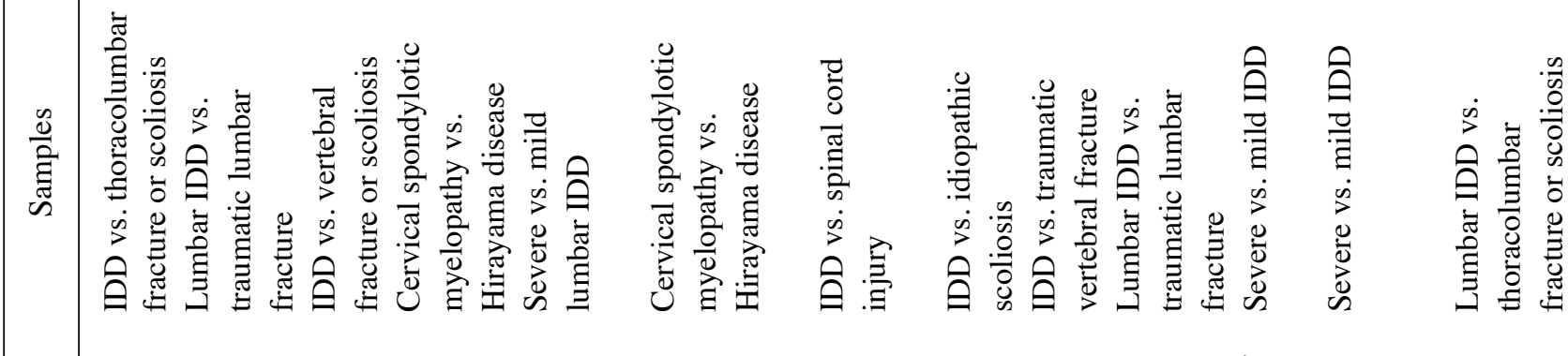

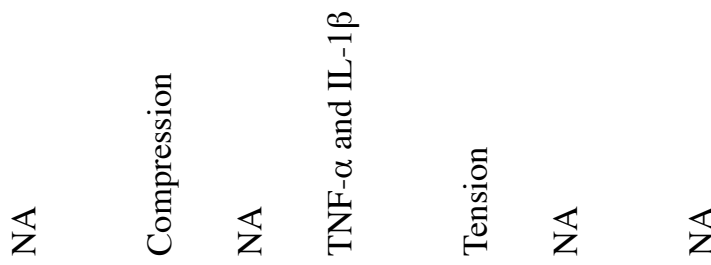

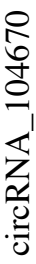

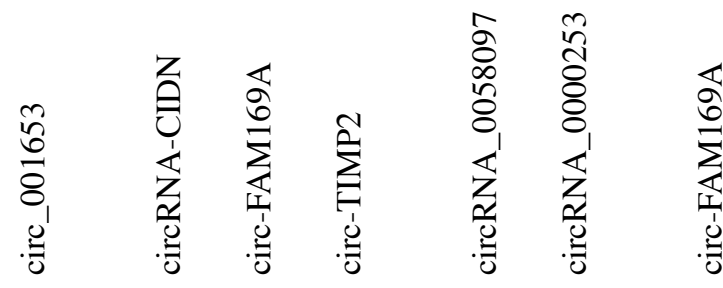

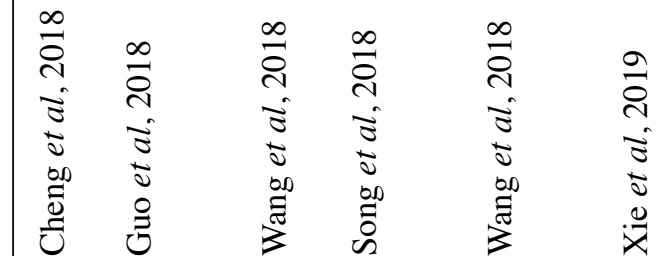

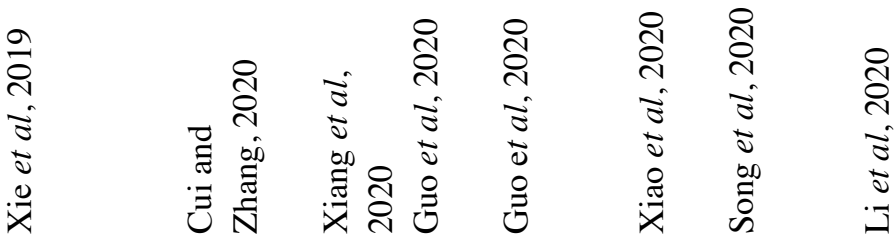

กิ่

$\Xi$

0
0
0
0
0
0
0
0
0
0
0
0
0
0
0
0
0
0
0
0
0
0 


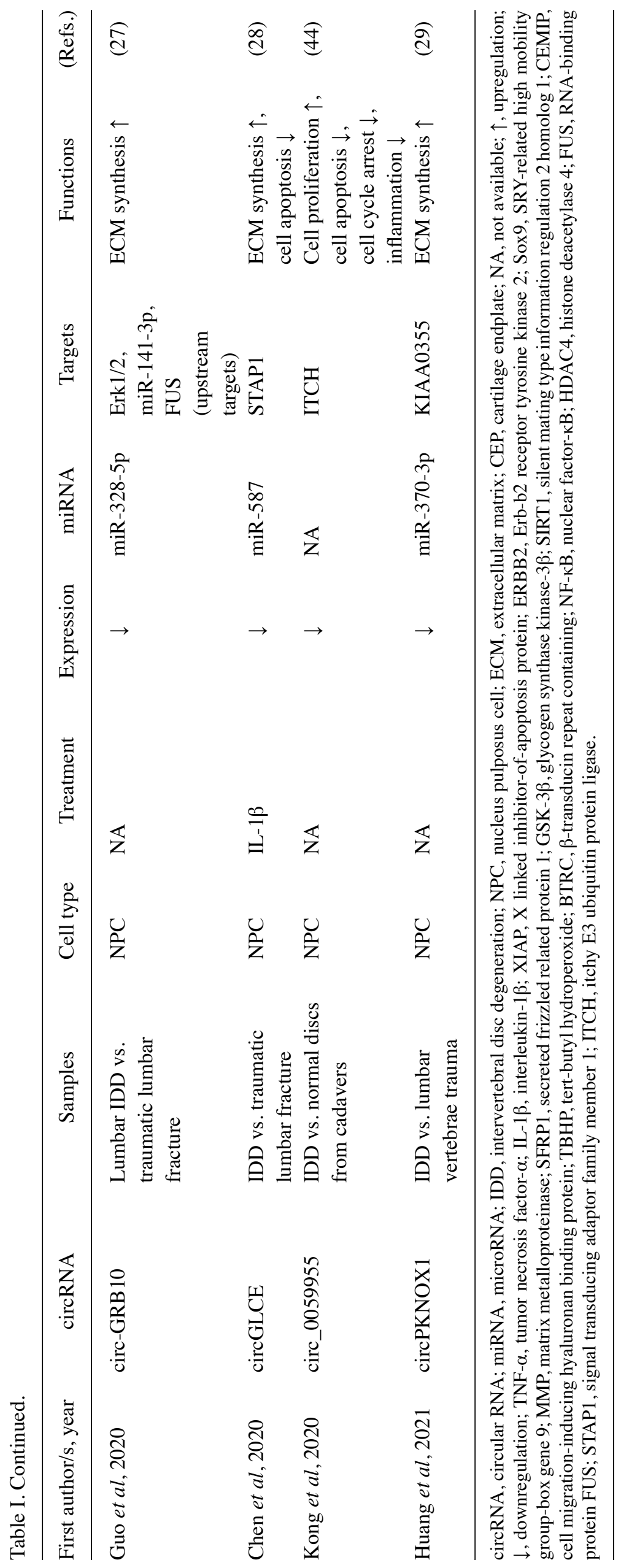




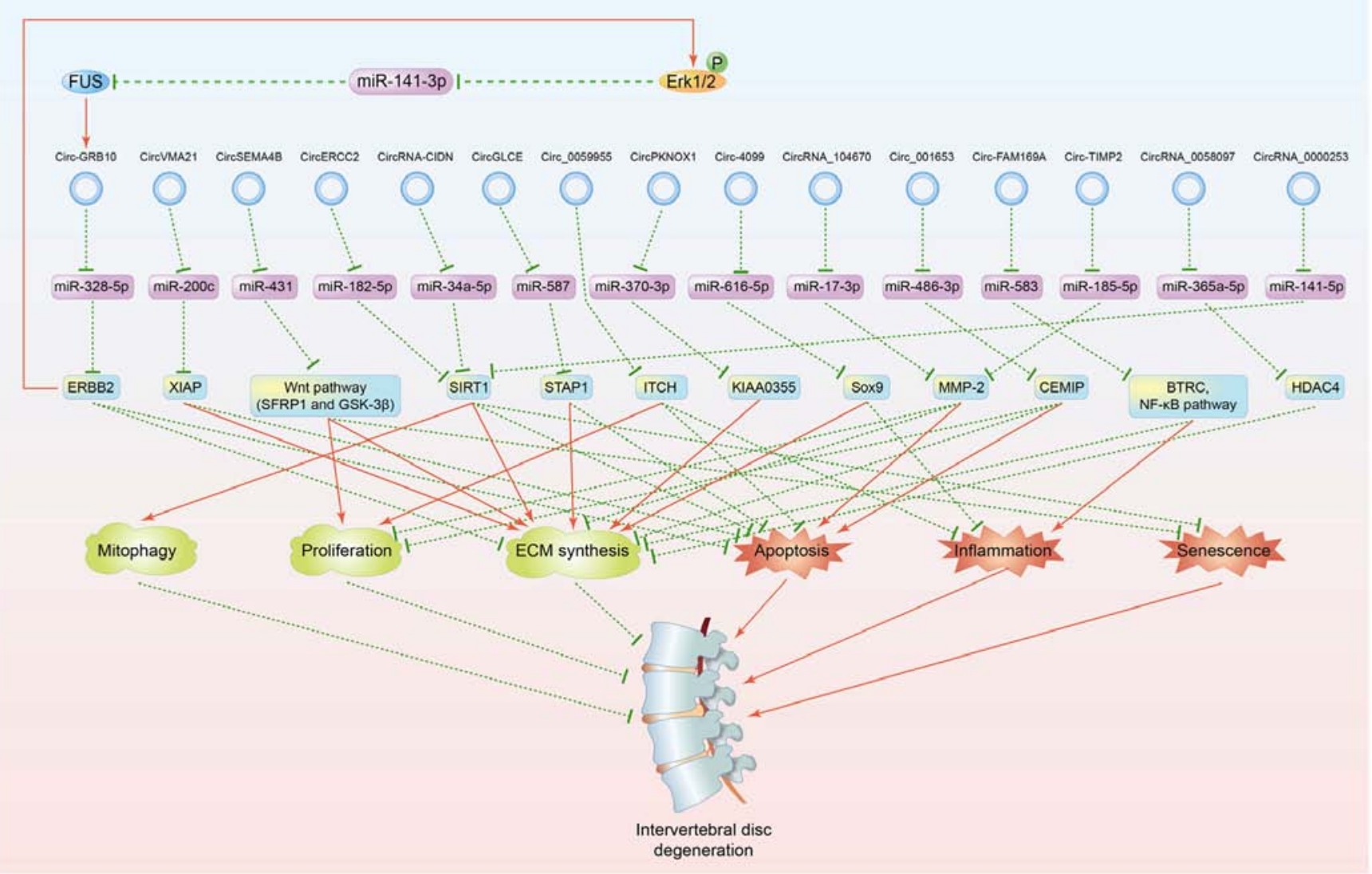

Figure 1. circRNAs involved in the regulation of IDD. Red solid lines indicate upregulation and green dashed lines represent downregulation. circRNA, circular RNA; miR, microRNA; P, phosphorylation; ECM, extracellular matrix; IDD, intervertebral disc degeneration; FUS, RNA-binding protein FUS; ERBB2, Erb-b2 receptor tyrosine kinase 2; XIAP, X linked inhibitor-of-apoptosis protein; SFRP1, secreted frizzled related protein 1; GSK-3 $\beta$, glycogen synthase kinase-3 $\beta$; SIRT1, silent mating type information regulation 2 homolog 1; STAP1, signal transducing adaptor family member 1; ITCH, itchy E3 ubiquitin protein ligase; Sox9, SRY-related high mobility group-box gene 9; MMP-2, matrix metalloproteinase 2; CEMIP, cell migration-inducing hyaluronan

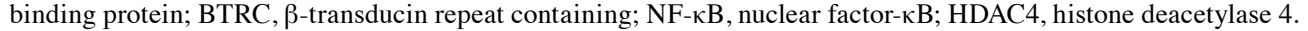

ECM metabolism by sponging miR-365a-5p and activating the expression of histone deacetylase 4 (HDAC4) in endplate chondrocytes induced by tension loading (Fig. 2).

We previously focused on the roles of exosome-transported circRNAs in IDD (37) and found that degenerative NPCs secreted more exosomes than the controls in NPC culture medium. Silencing circRNA_0000253, which was strongly upregulated in degenerative NPC exosomes, promoted ECM synthesis. Further investigations were performed both in vitro and in vivo, and indicated that exosome-transported circRNA_0000253 aggravates IDD via the miRNA-141-5p-SIRT1 axis (Fig. 3).

Collectively, circRNA-miRNA-mRNA axes play crucial roles in regulating ECM homeostasis.

\section{Roles of circRNA in cell proliferation and apoptosis}

The intervertebral disc, as the largest avascular tissue in the human body, is a cartilaginous structure with low cell density, despite the cells in the intervertebral disc being responsible for ECM synthesis and maintenance. IDD is characterized by a diminution in cell number and viability. Thus, cell proliferation and apoptosis are crucial to the pathogenesis of IDD $(38,39)$. For example, it was found that circVMA21 overexpression attenuated cell apoptosis in NPCs after treatment with TNF- $\alpha$ and IL- $1 \beta$ by acting as an miR-200c sponge and upregulating
XIAP (23). Urban et al (40) clarified the strong association between nutrition and IDD. It has been demonstrated that loss of nutrient supply can lead to cell death and an increase in ECM degradation, and hence to IDD (41). Guo et al (26) reported that circ-GRB10 expression was significantly downregulated and miR-328-5p expression was significantly upregulated in IDD samples compared with normal controls. Upregulation of circ-GRB10 expression significantly suppressed cell apoptosis in NPCs after nutrient deprivation in vitro. Further experiments demonstrated that circ-GRB10 serves as a regulator of NPC survival by sequestering miR-328-5p activity and subsequently promoting the expression of ERBB2. Li et al (42) found that circ-FAM169A expression was strongly upregulated in IDD tissues. Dual luciferase reporter assays corroborated the direct binding among circ-FAM169A, miR-583 and Sox9. Although functional and rescue experiments were lacking, functional enrichment analyses suggested that the circ-FAM169A-miR-583 axis might be involved in ECM metabolism and NPC survival.

In our previous study, interfering with circRNA_104670 facilitated the proliferation and suppressed the apoptosis of NPCs, as evaluated by MTT assays and cell flow cytometry (31). Wnt/ $\beta$-catenin signaling is involved in a multitude of biological processes, including cell proliferation, apoptosis and differentiation, embryonic development and tissue organization (43). It was reported that upregulation of circSEMA4B expression decreased 


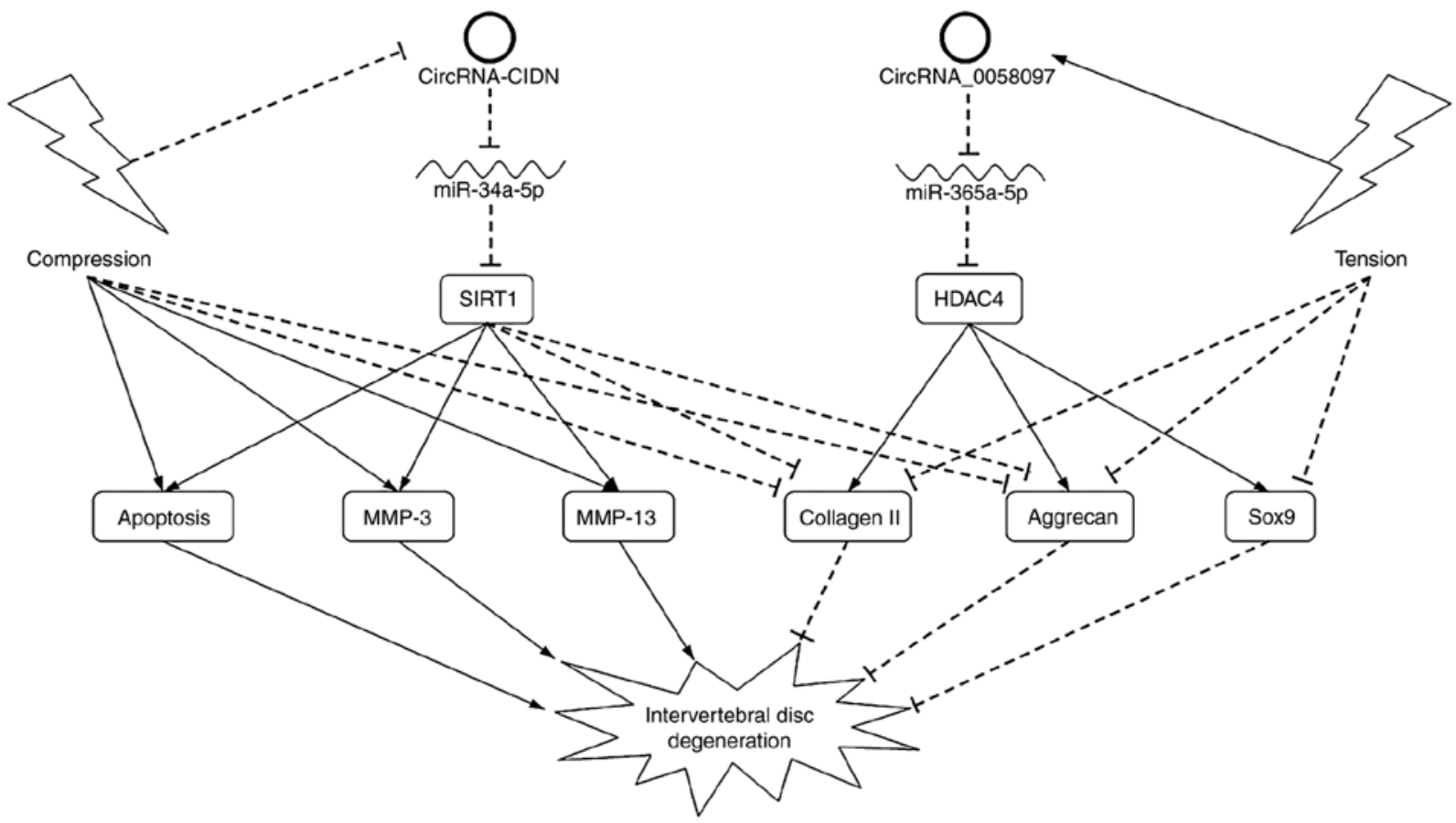

Figure 2. Mechanical loading induces intervertebral disc degeneration via circRNAs. circRNA, circular RNA; miR, microRNA; HDAC4, histone deacetylase 4; SIRT1, silent mating type information regulation 2 homolog 1; MMP, matrix metalloproteinase; collagen II, type II collagen; Sox9, SRY-related high mobility group-box gene 9 .

proliferation and activated senescence through the miR-431-Wnt pathway in NPCs after treatment with IL-1 $\beta$ (24). circ_001653 inhibition promoted proliferation and suppressed apoptosis in NPCs via miR-486-3p-CEMIP (32). circRNA-CIDN overexpression inhibited apoptosis via the miR-34a-5p-SIRT1 axis in NPCs after compression loading (33). Our previous study showed that exosome-transported circRNA_0000253 could activate apoptosis while decreasing the proliferation of NPCs by competitively absorbing miRNA-141-5p (37). It was found that circGLCE inhibition increased the rate of apoptosis in NPCs after IL-1 $\beta$ treatment by acting as a sponge for miR-587 (28).

Notably, Kong et al (44) focused on the interaction between circRNA and target protein rather than the extensively studied classic ceRNA mechanisms. This study found that the expression level of hsa_circ_0059955 was significantly lower in IDD specimens than that in control specimens. Knockdown of hsa_circ_0059955 inhibited proliferation and induced apoptosis and $G_{0} / G_{1}$ phase arrest in NPCs. The expression of itchy E3 ubiquitin protein ligase (ITCH) was negatively correlated with hsa_circ_0059955.Forced expression of ITCH counteracted the suppression of NPC proliferation induced by hsa_circ_0059955 inhibition. These results suggested the existence of the hsa circ_0059955-ITCH axis. However, further evidence verifying the direct binding between hsa_circ_0059955 and the ITCH protein is needed to make the hypothesis more convincing.

Thus, the aforementioned studies suggest that different circRNAs could exert opposing effects on NPC proliferation and apoptosis through ceRNA mechanisms.

\section{Roles of circRNA in inflammation}

Inflammatory mediators and signaling pathways are recognized as major contributors to the onset and development of IDD.
Elevated levels of inflammatory molecules have been detected in IDD tissues compared with those in healthy controls (45). Furthermore, ICs, particularly TNF- $\alpha$ and IL-1 $\beta$, have been demonstrated to stimulate and deteriorate the progression of IDD (46). Thus, treatment of NPCs with ICs has been widely applied to construct in vitro IDD models $(23,24,28,30,35)$. The nuclear factor- $\kappa \mathrm{B}(\mathrm{NF}-\kappa \mathrm{B})$ signaling pathway is closely implicated in numerous complex biological processes, such as immunity and inflammatory responses. There are two distinct pathways leading to the activation of the $N F-\kappa B$ signaling cascades. The canonical pathway is mediated by nuclear translocation of RelA (p65), c-Rel and p50, while the non-canonical pathway is mediated by nuclear translocation of p52 and RelB (47). It has been identified that the NF- $\kappa \mathrm{B}$ signaling pathway is a master regulator of inflammation and catabolism in IDD (48).

In the study by Wang et al (30), an IDD NPC model was generated by TNF- $\alpha$ treatment, and a higher expression level of circ-4099 was observed. When pathway inhibitors of the mitogen-activated protein kinase (MAPK) and NF- $\kappa$ B pathways were applied, the upregulation of circ-4099 expression induced by TNF- $\alpha$ was reversed, indicating that TNF- $\alpha$ enhanced circ-4099 expression via the MAPK and NF- $\kappa$ B pathways. Moreover, circ-4099 overexpression significantly decreased the expression level of IL-1 $\beta$, TNF- $\alpha$ and prostaglandin E2 in NPCs, while miR-616-5p mimics could impair the anti-inflammatory effects of circ-4099.

As a component of the Skp-Cullin-F-box-containing E3 ubiquitin ligase complex, BTRC recognizes the NF- $\kappa \mathrm{B}$ inhibitor $\mathrm{I} \kappa \mathrm{B} \alpha$ and precursor p100 for proteasomal degradation and processing, respectively. Therefore, BTRC plays an important role in both the canonical and non-canonical NF- $\kappa \mathrm{B}$ activation pathways (49). Guo et al (34) reported that circ-FAM169A was 


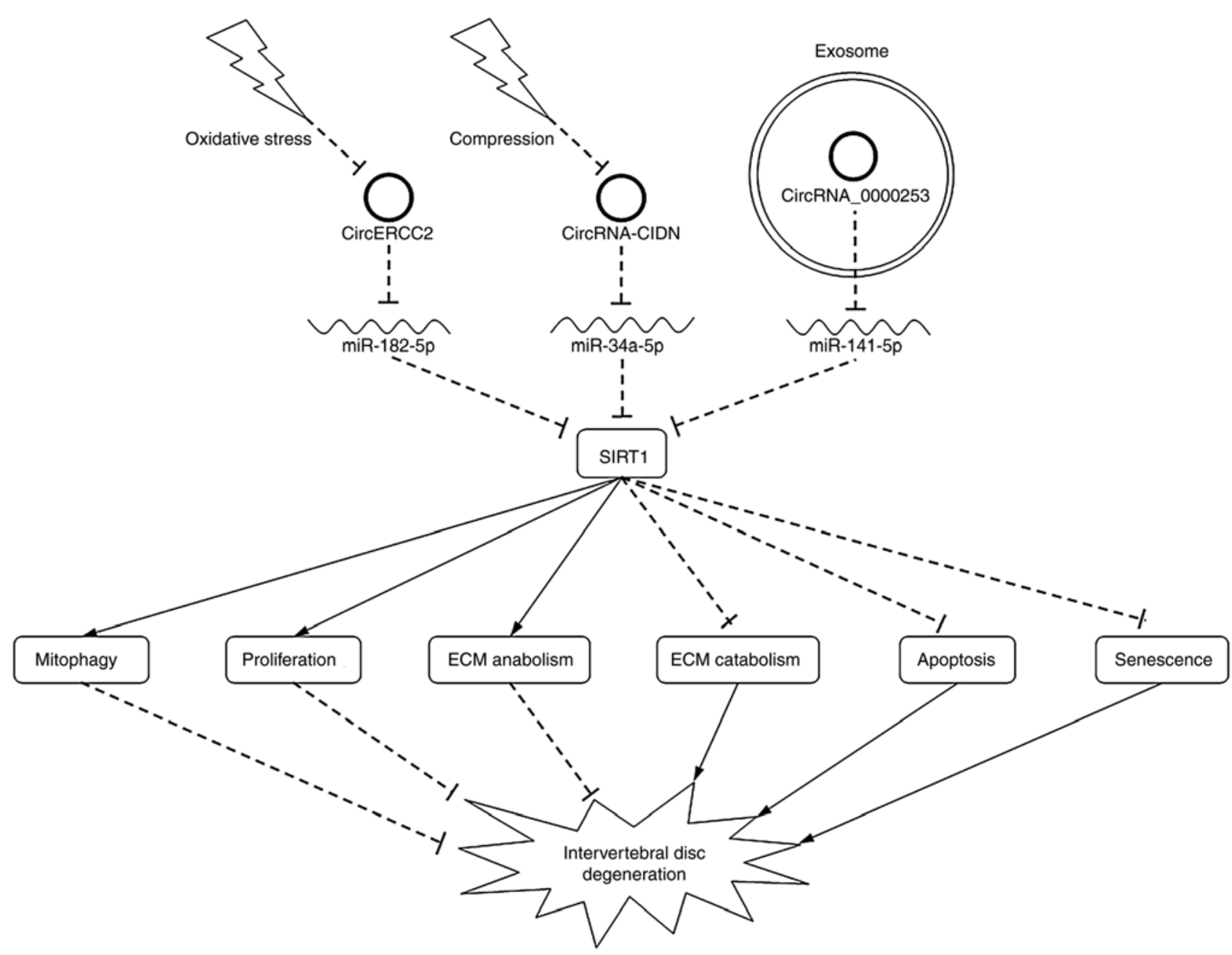

Figure 3. SIRT1 plays a protective role in intervertebral disc degeneration. circRNA, circular RNA; miR, microRNA; P, phosphorylation; ECM, extracellular matrix; SIRT1, silent mating type information regulation 2 homolog 1.

upregulated in IDD tissues. Knockdown of circ-FAM169A not only alleviated ECM degradation but also inhibited IL-1 $\beta$, TNF- $\alpha$ and IKB $\alpha$ expression, while circ-FAM169A overexpression exhibited the opposite effects. Silencing BTRC could reverse the adverse effects of circ-FAM169A. In addition, luciferase reporter assays confirmed the direct binding among circ-FAM169A, miR-583 and BTRC.

\section{Roles of circRNA in senescence}

Cellular senescence is characterized by irreversible proliferative arrest in response to multiple stresses, such as telomere dysfunction, DNA damage, organelle stress and oncogene activation (50). A growing body of evidence has indicated that cellular senescence participates in the pathological process of a number of chronic age-associated diseases, including IDD (51-54). To date, the detection of senescent cells depends on a combination of various markers, including senescence-associ ated- $\beta$-galactosidase (SA- $\beta$-gal), aberrant cell morphology, the nucleoside analogs p21 and p16, nuclear senescence-associated heterochromatin foci and components of the senescence-associated secretory phenotype (55). Previous studies identified that circSEMA4B and circERCC2 both inhibited cellular senescence, as determined using SA- $\beta$-gal staining, in NPCs stimulated by IL-1 $\beta$ and tert-butyl hydroperoxide (TBHP), respectively $(24,25)$. Hence, circRNAs could affect NPC senescence through miRNA-mRNA pathways.

\section{Roles of circRNA in mitophagy}

Mitochondria are double membrane-enclosed organelles, playing pivotal roles in energy production, reactive oxygen species (ROS) generation and cell death (56). Mitochondrial dysfunction is associated with the occurrence and development of various diseases. Therefore, dynamic and relatively stable control of mitochondrial quantity and quality is crucial for preserving the physiological functions of cells (57). Mitophagy, a special form of autophagy, is a conserved and essential process to maintain mitochondrial homeostasis by selectively eliminating dysfunctional mitochondria (56).

The PTEN induced kinase 1 (PINK1)/Parkin-mediated mitophagy pathway is a classical and well-studied pathway in degenerative diseases (58). Wang et al (59) discovered that PINK1 expression was upregulated in IDD tissues. NPCs were treated with $\mathrm{H}_{2} \mathrm{O}_{2}$ to induce oxidative stress, which aggravated mitochondrial dysfunction and cellular senescence, and activated mitophagy in NPCs. Silencing PINK1 enhanced oxidative stress-induced NPC senescence by inhibiting 
PINK1/Parkin-mediated mitophagy. Zhang et al (60) reported that Parkin expression was elevated both in IDD specimens and NPCs stimulated by TNF- $\alpha$. Knockdown of Parkin increased mitochondrial damage, ROS production and apoptosis in NPCs, while upregulating Parkin expression alleviated the progression of IDD via Parkin-mediated mitophagy. Wang et al (61) revealed that SIRT1 attenuated ROS accumulation, mitochondrial injury and cellular senescence through PINK1-dependent mitophagy in NPCs treated with high-magnitude compression. Therefore, mitophagy appears to be closely correlated with IDD development.

Hirayama disease is a special neurological disorder, predominantly affecting male adolescents. Thus, non-degenerative NP tissues could be obtained from patients undergoing anterior cervical discectomy and fusion due to this disease (62). We previously showed that circERCC2 expression was significantly downregulated in IDD tissues compared with that in Hirayama disease samples (25). Forced expression of circERCC2 decreased the rate of apoptosis and activated mitophagy, which was evaluated by detecting the key markers for mitophagy (PINK1, Parkin, p62 and LC3II/I ratio), in NPCs induced by TBHP in vitro. The direct binding between circERCC2 and miR-1825p, and between miR182-5p and SIRT1 was confirmed by dual-luciferase assays. Further analysis was performed and determined that circERCC2 alleviates IDD by regulating NPC apoptosis and mitophagy through the miR-182-5p-SIRT1 axis.

\section{Limitations and future directions}

Over the past few years, a growing number of studies have shown that numerous circRNAs can contribute to the development and progression of IDD, particularly NPC phenotypic modulation (Fig. 1). circRNAs are abundant, highly conserved and stable, with tissue- and development-specific expression patterns. Therefore, the in-depth and reliable elucidation of the association between circRNAs and IDD will eventually facilitate the establishment of novel diagnostic and treatment methods.

However, there are still some limitations in the available studies. First, to the best of our knowledge, 17 studies have been published that investigated the potential roles of circRNAs in IDD. Of these, 15 mainly focused on ceRNA mechanisms, indicating that circRNAs exert biological functions by acting as miRNA sponges, and established circRNA-miRNA-mRNA axes. Notably, Kong et al (44) attempted to study the interaction between circRNA and target protein instead of classic ceRNA mechanisms. The results suggested that hsa_circ_0059955 inhibition negatively regulated NPC survival via the ITCH protein. However, this finding was not confirmed, as experiments verifying the direct binding between hsa_circ_0059955 and ITCH protein were not conducted. Notably, circRNAs not only sponge and sequester proteins, but also act as protein scaffolds to mediate the formation of circRNA-protein complexes and the cellular localization of specific proteins (9). Notably, although circRNAs are generally considered to be non-coding, as they lack 5' caps and polyadenylated tails that are essential for translation initiation, some circRNAs can function as templates for protein synthesis through cap-independent translation driven by internal ribosome entry sites or N6-methyladenosine (9).
Accordingly, future studies are required to discover other functional mechanisms of circRNAs in IDD in addition to their serving as miRNA sponges.

Second, most of the present studies concentrated on the downstream mechanisms of circRNAs, but the question of what causes the aberrantly altered expression of circRNAs in IDD has been less discussed. Notably, studies by Guo et al $(26,27)$ established a complex signaling circuitry of circ-GBR10 rather than a linear pathway in IDD. It was found that an RNA-binding protein, FUS, was an upstream regulator of circ-GRB10. RNA immunoprecipitation further confirmed the direct interaction between circ-GRB10 and the FUS protein. Combining the results of two published studies by Guo et al $(26,27)$, a circ-GBR10-m iR-328-5p-ERBB2-Erk1/2 phosphorylation-miR-141-3p-FUS loop was constructed. In the future, it will be worthwhile to fully investigate circRNAs with their upstream regulators and downstream targets to establish circRNA networks. Further elucidation of the crosstalk among circRNAs can help us to recognize the key nodes to improve diagnosis and treatment.

Third, IDD is an extremely complex process. Studies tend to focus on NPCs, as they are the main type of cells in the NP and are responsible for ECM production. However, IDD also includes the deterioration of both AF and CEPs. Xiao et al (36) focused on endplate chondrocytes instead of NPCs and demonstrated that inhibition of circRNA_0058097 could exert positive effects on endplate chondrocyte degeneration via the miR-365a-5p-HDAC4 axis. More studies are needed to reveal how circRNAs affect the IDD process by regulating the functions of cells in AF and CEPs. Furthermore, numerous studies simulated IDD by culturing NPCs with ICs, such as IL-1 $\beta$ and TNF- $\alpha$. In addition to ICs, oxidative stress, nutrient deprivation and mechanical loading are crucial factors inducing IDD (46,63-65). Xiang et al (33) indicated that circRNA-CIDN alleviated IDD induced by compression through the miR-34a-5p-SIRT1 axis. Thus, more research should be performed in different IDD models. In addition, proliferation, apoptosis, inflammation and ECM metabolism have been extensively studied. Only two studies described senescence $(24,25)$, and one study discussed mitophagy in IDD (25). Therefore, the important phenotypes mediated by circRNAs, including cellular senescence, autophagy, mitophagy and ferroptosis, in IDD have not been sufficiently illuminated $(50,57,66,67)$.

Recently, exosome-transported circRNAs have attracted increasing attention from scientists. Exosomes are small membrane vesicles that originate from multivesicular endosomes by inverse budding. Secreted by most eukaryotes, exosomes can carry various molecules, including lipids, proteins and nucleic acids, from one cell to another (68). It was previously noted that exosomal circRNA_0000253 can aggravate the progression of IDD via the miRNA-141-5p-SIRT1 axis (37), and the roles of exosome-transported circRNAs have become a hot topic in the research field of cancer (69). Therefore, it is essential to further determine whether these novel circRNAs are involved in the pathological process of IDD.

\section{Conclusion}

In conclusion, the latest evidence has confirmed that circRNAs play crucial roles in ECM synthesis and decomposition, 
inflammation, cellular proliferation, apoptosis, senescence and mitophagy, mainly by functioning as sponges for miRNAs in the pathogenesis of IDD.

\section{Acknowledgements}

Not applicable.

\section{Funding}

This study was supported by the General Program of National Natural Science Foundation of China (grant nos. 81871552, 81972093 and 82072488).

\section{Availability of data and materials}

Not applicable.

\section{Authors' contributions}

$\mathrm{ZL}, \mathrm{FZ}$ and $\mathrm{JJ}$ were responsible for the conceptualization of the study. FZ, JJ and FL were responsible for the study design, and ZL, FL, XM and XX for the search strategy, article inclusion and interpretation. All authors were involved in original draft preparation. ZL, FZ and JJ were responsible for reviewing and editing the manuscript, and FZ and JJ supervised the project. All authors have read and approved the manuscript. Data authentication is not applicable.

\section{Ethics approval and consent to participate}

Not applicable.

\section{Patient consent for publication}

Not applicable.

\section{Competing interests}

The authors declare that they have no competing interests.

\section{References}

1. Hartvigsen J, Hancock MJ, Kongsted A, Louw Q, Ferreira ML, Genevay S, Hoy D, Karppinen J, Pransky G, Sieper J, et al: What low back pain is and why we need to pay attention. Lancet 391: 2356-2367, 2018.

2. Cieza A, Causey K, Kamenov K, Hanson SW, Chatterji S and Vos T: Global estimates of the need for rehabilitation based on the Global Burden of Disease study 2019: A systematic analysis for the Global Burden of Disease Study 2019. Lancet 396: 2006-2017, 2021.

3. Walker BF: The prevalence of low back pain: A systematic review of the literature from 1966 to 1998. J Spinal Disord 13: 205-217, 2000.

4. Walker BF, Muller R and Grant WD: Low back pain in Australian adults: Prevalence and associated disability. J Manipulative Physiol Ther 27: 238-244, 2004.

5. Vlaeyen JW, Maher CG, Wiech K, Van Zundert J, Meloto CB, Diatchenko L, Battie MC, Goossens M, Koes B and Linton SJ: Low back pain. Nat Rev Dis Primers 4: 52, 2018.

6. Wise CA, Sepich D, Ushiki A, Khanshour AM, Kidane YH, Makki N, Gurnett CA, Gray RS, Rios JJ, Ahituv N and Solnica-Krezel L: The cartilage matrisome in adolescent idiopathic scoliosis. Bone Res 8: 13, 2020.
7. Adams MA and Roughley PJ: What is intervertebral disc degeneration, and what causes it?. Spine (Phila Pa 1976) 31: 2151-2161, 2006.

8. Risbud MV and Shapiro IM: Role of cytokines in intervertebral disc degeneration: Pain and disc content. Nat Rev Rheumatol 10: 44-56, 2014.

9. Chen LL: The expanding regulatory mechanisms and cellular functions of circular RNAs. Nat Rev Mol Cell Biol 21: 475-490, 2020.

10. Thomson DW and Dinger ME: Endogenous microRNA sponges: Evidence and controversy. Nat Rev Genet 17: 272-283, 2016.

11. Shen S, Wu Y, Chen J, Xie Z, Huang K, Wang G, Yang Y, Ni W, Chen Z, Shi P, et al: CircSERPINE2 protects against osteoarthritis by targeting miR-1271 and ETS-related gene. Ann Rheum Dis 78: 826-836, 2019.

12. Cai Y, Liang R, Xiao S, Huang Q, Zhu D, Shi GP, Ouyang Q and Yang M: Circ_0088194 promotes the invasion and migration of rheumatoid arthritis fibroblast-like synoviocytes via the miR-766-3p/MMP2 axis. Front Immunol 12: 628654, 2021.

13. Han S, Kuang M, Sun C, Wang H, Wang D and Liu Q: Circular RNA hsa_circ_0076690 acts as a prognostic biomarker in osteoporosis and regulates osteogenic differentiation of hBMSCs via sponging miR-152. Aging (Albany NY) 12: 15011-15020, 2020.

14. Wu Y, Xie Z, Chen J, Chen J, Ni W, Ma Y, Huang K, Wang G, Wang J, Ma J, et al: Circular RNA circTADA2A promotes osteosarcoma progression and metastasis by sponging miR-203a-3p and regulating CREB3 expression. Mol Cancer 18: 73, 2019.

15. Hao Y, Lu C, Zhang B, Xu Z, Guo H and Zhang G: CircPVT1 up-regulation attenuates steroid-induced osteonecrosis of the femoral head through regulating miR-21-5p-mediated Smad7/TGF $\beta$ signalling pathway. J Cell Mol Med 25: 4608-4622, 2021.

16. Chen C, Tan H, Bi J, Li Z, Rong T, Lin Y, Sun L, Li X and Shen J: Identification of competing endogenous RNA regulatory networks in vitamin A deficiency-induced congenital scoliosis by transcriptome sequencing analysis. Cell Physiol Biochem 48: 2134-2146, 2018

17. Peng $P$, Zhang B, Huang J, Xing C, Liu W, Sun C, Guo W, Yao S, Ruan W, Ning G, et al: Identification of a circRNA-miRNA-mRNA network to explore the effects of circRNAs on pathogenesis and treatment of spinal cord injury. Life Sci 257: 118039, 2020.

18. Li Z, Chen X, Xu D, Li S, Chan MT and Wu WK: Circular RNAs in nucleus pulposus cell function and intervertebral disc degeneration. Cell Prolif 52: e12704, 2019.

19. Li Y, Zhou S, Peng P, Wang X, Du L, Huo Z and Xu B: Emerging role of circular RNA in intervertebral disc degeneration: Knowns and unknowns (Review). Mol Med Rep 22: 3057-3065, 2020.

20. Le Maitre CL, Pockert A, Buttle DJ, Freemont AJ and Hoyland JA: Matrix synthesis and degradation in human intervertebral disc degeneration. Biochem Soc Trans 35: 652-655, 2007.

21. Binch ALA, Shapiro IM and Risbud MV: Syndecan-4 in intervertebral disc and cartilage: Saint or synner? Matrix Biol 52-54: 355-362, 2016.

22. Wang SZ, Rui YF, Lu J and Wang C: Cell and molecular biology of intervertebral disc degeneration: Current understanding and implications for potential therapeutic strategies. Cell Prolif 47: 381-390, 2014.

23. Cheng X, Zhang L, Zhang K, Zhang G, Hu Y, Sun X, Zhao C, $\mathrm{Li} \mathrm{H}, \mathrm{Li}$ YM and Zhao J: Circular RNA VMA21 protects against intervertebral disc degeneration through targeting miR-200c and X linked inhibitor-of-apoptosis protein. Ann Rheum Dis 77: 770-779, 2018.

24. Wang X, Wang B, Zou M, Li J, Lü G, Zhang Q, Liu F and Lu C: CircSEMA4B targets miR-431 modulating IL-1 $\beta$-induced degradative changes in nucleus pulposus cells in intervertebral disc degeneration via Wnt pathway. Biochim Biophys Acta Mol Basis Dis 1864: 3754-3768, 2018.

25. Xie L, Huang W, Fang Z, Ding F, Zou F, Ma X, Tao J, Guo J, Xia X, Wang H, et al: CircERCC2 ameliorated intervertebral disc degeneration by regulating mitophagy and apoptosis through miR-182-5p/SIRT1 axis. Cell Death Dis 10: 751, 2019.

26. Guo W, Zhang B, Mu K, Feng SQ, Dong ZY, Ning GZ, Li HR, Liu S, Zhao L, Li Y, et al: Circular RNA GRB10 as a competitive endogenous RNA regulating nucleus pulposus cells death in degenerative intervertebral disk. Cell Death Dis 9: 319, 2018.

27. Guo W, Mu K, Zhang B, Sun C, Zhao L, Li HR, Dong ZY and Cui Q: The circular RNA circ-GRB10 participates in the molecular circuitry inhibiting human intervertebral disc degeneration. Cell Death Dis 11: 612, 2020. 
28. Chen Z, Zhang W, Deng M, Li Y and Zhou Y: CircGLCE alleviates intervertebral disc degeneration by regulating apoptosis and matrix degradation through the targeting of miR-587/STAP1. Aging (Albany NY.) 12: 21971-21991, 2020.

29. Huang Y, Gao J, Wang J, Ye H, Yao T, Xu Y, Chen Z, Shen S and Ma J: Inhibition of intervertebral disc disease progression via the circPKNOX1-miR-370-3p-KIAA0355 axis. Cell Death Discov 7: 39, 2021.

30. Wang H, He P, Pan H, Long J, Wang J, Li Z, Liu H, Jiang W and Zheng Z: Circular RNA circ-4099 is induced by TNF- $\alpha$ and regulates ECM synthesis by blocking miR-616-5p inhibition of Sox9 in intervertebral disc degeneration. Exp Mol Med 50: 1-14, 2018.

31. Song J, Wang HL, Song KH, Ding ZW, Wang HL, Ma XS, Lu FZ, Xia XL, Wang YW, Fei-Zou and Jiang JY: CircularRNA_104670 plays a critical role in intervertebral disc degeneration by functioning as a ceRNA. Exp Mol Med 50: 1-12, 2018.

32. Cui S and Zhang L: Circ 001653 silencing promotes the proliferation and ECM synthesis of NPCs in IDD by downregulating miR-486-3p-mediated CEMIP. Mol Ther Nucleic Acids 20 385-399, 2020

33. Xiang Q, Kang L, Wang J, Liao Z, Song Y, Zhao K, Wang K, Yang $\mathrm{C}$ and Zhang Y: CircRNA-CIDN mitigated compression loading-induced damage in human nucleus pulposus cells via miR-34a-5p/SIRT1 axis. EBioMedicine 53: 102679, 2020.

34. Guo W, Mu K, Zhang B, Sun C, Zhao L, Dong ZY and Cui Q: The circular RNA FAM169A functions as a competitive endogenous RNA and regulates intervertebral disc degeneration by targeting miR-583 and BTRC. Cell Death Dis 11: 315, 2020.

35. Guo W, Zhang B, Sun C, Duan HQ, Liu WX, Mu K, Zhao L, Li HR, Dong ZY and Cui Q: Circular RNA derived from TIMP2 functions as a competitive endogenous RNA and regulates intervertebral disc degeneration by targeting miR-185-5p and matrix metalloproteinase 2. Int J Mol Med 46: 621-632, 2020.

36. Xiao L, Ding B, Xu S, Gao J, Yang B, Wang J and $\mathrm{Xu} \mathrm{H}$ CircRNA_0058097 promotes tension-induced degeneration of endplate chondrocytes by regulating HDAC4 expression through sponge adsorption of miR-365a-5p. J Cell Biochem 121: 418-429, 2020.

37. Song J, Chen ZH, Zheng CJ, Song KH, Xu GY, Xu S, Zou F, Ma XS, Wang HL and Jiang JY: Exosome-Transported circRNA_0000253 competitively adsorbs MicroRNA-141-5p and increases IDD. Mol Ther Nucleic Acids 21: 1087-1099, 2020.

38. Ding F, Shao ZW and Xiong LM: Cell death in intervertebral disc degeneration. Apoptosis 18: 777-785, 2013.

39. Zhao CQ, Jiang LS and Dai LY: Programmed cell death in intervertebral disc degeneration. Apoptosis 11: 2079-2088, 2006.

40. Urban JP, Smith S and Fairbank JC: Nutrition of the intervertebral disc. Spine (Phila Pa 1976) 29: 2700-2709, 2004.

41. Grunhagen T, Wilde G, Soukane DM, Shirazi-Adl SA and Urban JP: Nutrient supply and intervertebral disc metabolism. J Bone Joint Surg Am 88 (Suppl 2): S30-S35, 2006.

42. Li Y, Pan D, Liu S, Xing X, Zhou H, Zhang B, Zhang D, Li B, Li G, Tao B, et al: Identification of circ-FAM169A sponges miR-583 involved in the regulation of intervertebral disc degeneration. J Orthop Translat 26: 121-131, 2020.

43. Kahn M: Can we safely target the WNT pathway? Nat Rev Drug Discov 13: 513-532, 2014

44. Kong D, Gu R, Zhang C and Yin R: Knockdown of hsa circ_0059955 induces apoptosis and cell cycle arrest in nucleus pulposus cells via inhibiting itchy E3 ubiquitin protein ligase. Drug Des Devel Ther 14: 3951-3963, 2020.

45. Lyu FJ, Cui H, Pan H, Mc Cheung K, Cao X, Iatridis JC and Zheng Z: Painful intervertebral disc degeneration and inflammation: From laboratory evidence to clinical interventions. Bone Res 9: 7, 2021.

46. Wang Y, Che M, Xin J, Zheng Z, Li J and Zhang S: The role of IL-1beta and TNF-alpha in intervertebral disc degeneration. Biomed Pharmacother 131: 110660, 2020

47. Miraghazadeh B and Cook MC: Nuclear factor-kappaB in autoimmunity: Man and Mouse. Front Immunol 9: 613, 2018.

48. Zhang GZ, Liu MQ, Chen HW, Wu ZL, Gao YC, Ma ZJ, He XG and Kang XW: NF- $\mathrm{KB}$ signalling pathways in nucleus pulposus cell function and intervertebral disc degeneration. Cell Prolif 54 e13057, 2021

49. Shi M, Cho H, Inn KS, Yang A, Zhao Z, Liang Q, Versteeg GA, Amini-Bavil-Olyaee S, Wong LY, Zlokovic BV, et al: Negative regulation of NF- $\kappa B$ activity by brain-specific TRIpartite Motif protein 9. Nat Commun 5: 4820, 2014.
50. Zhang Y, Yang B, Wang J, Cheng F, Shi K, Ying L, Wang C, Xia K, Huang X, Gong Z, et al: Cell senescence: A nonnegligible cell state under survival stress in pathology of intervertebral disc degeneration. Oxid Med Cell Longev 2020: 9503562, 2020.

51. Baker DJ and Petersen RC: Cellular senescence in brain aging and neurodegenerative diseases: Evidence and perspectives. J Clin Invest 128: 1208-1216, 2018.

52. Grootaert MOJ, Moulis M, Roth L, Martinet W, Vindis C, Bennett MR and De Meyer GRY: Vascular smooth muscle cell death, autophagy and senescence in atherosclerosis. Cardiovasc Res 114: 622-634, 2018.

53. Lee $\mathrm{S}$ and Schmitt CA: The dynamic nature of senescence in cancer. Nat Cell Biol 21: 94-101, 2019.

54. Wang F, Cai F, Shi R, Wang XH and Wu XT: Aging and age related stresses: A senescence mechanism of intervertebral disc degeneration. Osteoarthritis Cartilage 24: 398-408, 2016.

55. Di Micco R, Krizhanovsky V, Baker D and d'Adda di Fagagna F: Cellular senescence in ageing: From mechanisms to therapeutic opportunities. Nat Rev Mol Cell Biol 22: 75-95, 2021.

56. Youle RJ and Narendra DP: Mechanisms of mitophagy. Nat Rev Mol Cell Biol 12: 9-14, 2011.

57. Sun K, Jing X, Guo J, Yao X and Guo F: Mitophagy in degenerative joint diseases. Autophagy: Sep 24, 2020 (Epub ahead of print). doi: 10.1080/15548627.2020.1822097.

58. Doblado L, Lueck C, Rey C, Samhan-Arias AK, Prieto I, Stacchiotti A and Monsalve M: Mitophagy in human diseases. Int J Mol Sci 22: 3903, 2021.

59. Wang Y, Shen J, Chen Y, Liu H, Zhou H, Bai Z, Hu Z and Guo X: PINK1 protects against oxidative stress induced senescence of human nucleus pulposus cells via regulating mitophagy. Biochem Biophys Res Commun 504: 406-414, 2018.

60. Zhang Z, Xu T, Chen J, Shao Z, Wang K, Yan Y, Wu C, Lin J, Wang $\mathrm{H}$, Gao W, et al: Parkin-mediated mitophagy as a potential therapeutic target for intervertebral disc degeneration. Cell Death Dis 9: 980, 2018.

61. Wang Y, Wang H, Zhuo Y, Hu Y, Zhang Z, Ye J, Liu L, Luo L, Zhao C, Zhou Q and Li P: SIRT1 alleviates high-magnitude compression-induced senescence in nucleus pulposus cells via PINK1-dependent mitophagy. Aging (Albany NY) 12 16126-16141, 2020

62. Lyu F, Zheng C, Wang H, Nie C, Ma X, Xia X, Zhu W, Jin X, $\mathrm{Hu}$ Y, Sun Y, et al: Establishment of a clinician-led guideline on the diagnosis and treatment of Hirayama disease using a modified Delphi technique. Clin Neurophysiol 131: 1311-1319, 2020.

63. Wang XB, Wang H, Long HQ, Li DY and Zheng X: LINC00641 regulates autophagy and intervertebral disc degeneration by acting as a competitive endogenous RNA of miR-153-3p under nutrition deprivation stress. J Cell Physiol 234: 7115-7127, 2019.

64. Dai S, Liang T, Shi X, Luo Z and Yang H: Salvianolic acid B protects intervertebral discs from oxidative stress-induced degeneration via activation of the JAK2/STAT3 signaling pathway. Oxid Med Cell Longev 2021: 6672978, 2021.

65. He R, Wang Z, Cui M, Liu S, Wu W, Chen M, Wu Y, Qu Y, Lin H, Chen S, et al: HIF1A Alleviates compression-induced apoptosis of nucleus pulposus derived stem cells via upregulating autophagy. Autophagy: Jan 18, 2021 (Epub ahead of print). doi: 10.1080/ 15548627.2021 .1872227

66. Lan T, Shiyu-Hu, Shen Z, Yan B and Chen J: New insights into the interplay between miRNAs and autophagy in the aging of intervertebral discs. Ageing Res Rev 65: 101227, 2021

67. Yang RZ, Xu WN, Zheng HL, Zheng XF, Li B, Jiang LS and Jiang SD: Involvement of oxidative stress-induced annulus fibrosus cell and nucleus pulposus cell ferroptosis in intervertebral disc degeneration pathogenesis. J Cell Physiol 236: 2725-2739, 2021

68. Wang Y, Liu J, Ma J, Sun T, Zhou Q, Wang W, Wang G, Wu P, Wang H, Jiang L, et al: Exosomal circRNAs: Biogenesis, effect and application in human diseases. Mol Cancer 18: 116, 2019.

69. Seimiya T, Otsuka M, Iwata T, Shibata C, Tanaka E, Suzuki T and Koike K: Emerging roles of exosomal circular RNAs in cancer. Front Cell Dev Biol 8: 568366, 2020.

This work is licensed under a Creative Commons Attribution-NonCommercial-NoDerivatives 4.0 International (CC BY-NC-ND 4.0) License. 